\title{
Intrageneric diversity of type-I ketosynthase domain genes in the genus Nocardia
}

\author{
Hisayuki Komaki, Tomohiko Tamura and Ken-ichiro Suzuki \\ The Journal of Antibiotics (2011) 64, 617-620; doi:10.1038/ja.2011.42; published online 18 May 2011
}

Keywords: ketosynthase domain; Nocardia; polyketide synthase

Nocardia species are the causative agents of nocardiosis in humans and are known to produce a variety of bioactive compounds with antimicrobial, antitumor and immunosuppressive activities. Nocardia brasiliensis is often reported to produce various bioactive compounds such as indole alkaloid, terpenoid, peptide and aromatic compounds. ${ }^{1}$ Other species, including $N$. asteroides and $N$. farcinica, are not widely reported to be producers of bioactive compounds, and their products are generally siderophores. ${ }^{2,3}$ Reports regarding the production of polyketide compounds, such as macrolides and polyethers, synthesized by modular type-I polyketide synthases (PKS-Is), are limited to a few species such as $N$. brasiliensis and $N$. terpenica, ${ }^{4-7}$ although some polyketide-producing Nocardia strains are yet to be identified at the species level. ${ }^{8}$ The recent $N$. farcinica IFM 10152 genome sequencing project revealed the presence of only six PKS-I genes, suggesting that PKS-I genes are less abundant in N. farcinica compared with Streptomyces spp. ${ }^{9-11} \mathrm{Nfa7640}(n b t B)$ and nfa7650 ( nbtC) are PKS-I genes in a mixed PKS/non-ribosomal peptide synthetase (NRPS) gene cluster involved in the synthesis of siderophores named nocobactins; ${ }^{2}$ however, metabolites of the other four PKS-I genes, nfa1890, nfa30250, nfa43240 and nfa55930, are still unclear, although nfa30250 is predicted to be mycocerosic acid synthase. Because none of these four genes organizes multimodular PKS-I gene clusters, their metabolites are not likely to be structurally complex and the strain will not produce typical polyketide compounds, such as macrolides and polyethers. This may be one reason why type-I polyketide compounds are not frequently produced by Nocardia. The biosynthetic potential of actinomycetes is often approximated through the analysis of genes involved in synthesis of secondary metabolites, such as PKS-Is, type-II PKSs and NRPSs. ${ }^{12-15}$ However, the diversity and novelty of these genes in Nocardia species other than N. farcinica have not been studied. There is no information regarding the relationship between taxonomy and gene distribution. In our study, we focused on PKS-I genes and investigated their relationship with Nocardia taxonomy. This allowed us to assess their ability to produce various type-I polyketide compounds.
The strains evaluated included 24 Nocardia strains belonging to 22 species (Figure 1). Genomic DNA was prepared using a DNeasy Blood \& Tissue Kit (QIAGEN Sciences, Germantown, MD, USA). Ketosynthase (KS) domain DNA regions in PKS-I genes were amplified as reported previously. ${ }^{15}$ The polymerase chain reaction (PCR) products were cloned using a TOPO TA Cloning Kit for Sequencing (Invitrogen, Carlsbad, CA, USA) and transformed into ECOS competent Escherichia coli DH5 $\alpha$ (Nippon Gene, Toyama, Japan). Approximately 10 recombinant $E$. coli clones were picked for each PCR sample and the inserted DNA segments were amplified directly from the cells by colony PCR with M13 forward (5'-GTAAAACGACGGCCAG- $3^{\prime}$ ) and M13 reverse (5'-CAGGAAACAGCTATGAC- $\left.3^{\prime}\right)$ primers. The amplified inserts were purified using the Agencourt AMPure (Beckman Coulter Genomics, Danvers, MA, USA). DNA sequencing of the inserts was performed with a BigDye Terminator v3.1 Cycle Sequencing Kit (Applied Biosystems Japan Ltd., Tokyo, Japan), M13 primers and a 3130xl Genetic Analyzer (Applied Biosystems Japan Ltd.). The obtained sequences were queried with the BLASTX software program (http://blast.ncbi.nlm.nih.gov/Blast.cgi). The deduced amino-acid sequences were aligned, and a phylogenetic tree was constructed using Mega $4^{16}$ and the neighbor-joining method.

PKS-I genes were successfully amplified from all test strains. Because PKS-Is are large multimodular enzymes, and even a single strain generally possesses several PKS-I genes in its genome, the amplicon from each strain contained several types of KS sequences. We cloned the PCR products and determined 225 DNA sequences (approximately $1100 \mathrm{bp}$ each) from the 24 strains. After removing duplicate sequences, 107 non-redundant sequences were subjected to a BLAST search. All sequences showed low similarities $(<78 \%)$ to PKSI genes reported to be involved in the synthesis of known polyketide compounds and/or revealed by whole genome sequencing projects, although 50 sequences among them showed over $80 \%$ identity to Nocardia PKS-I genes whose products are yet to be identified (Supplementary Table 1). To determine the diversity of the 107 sequences, the deduced amino-acid sequences were compared using 


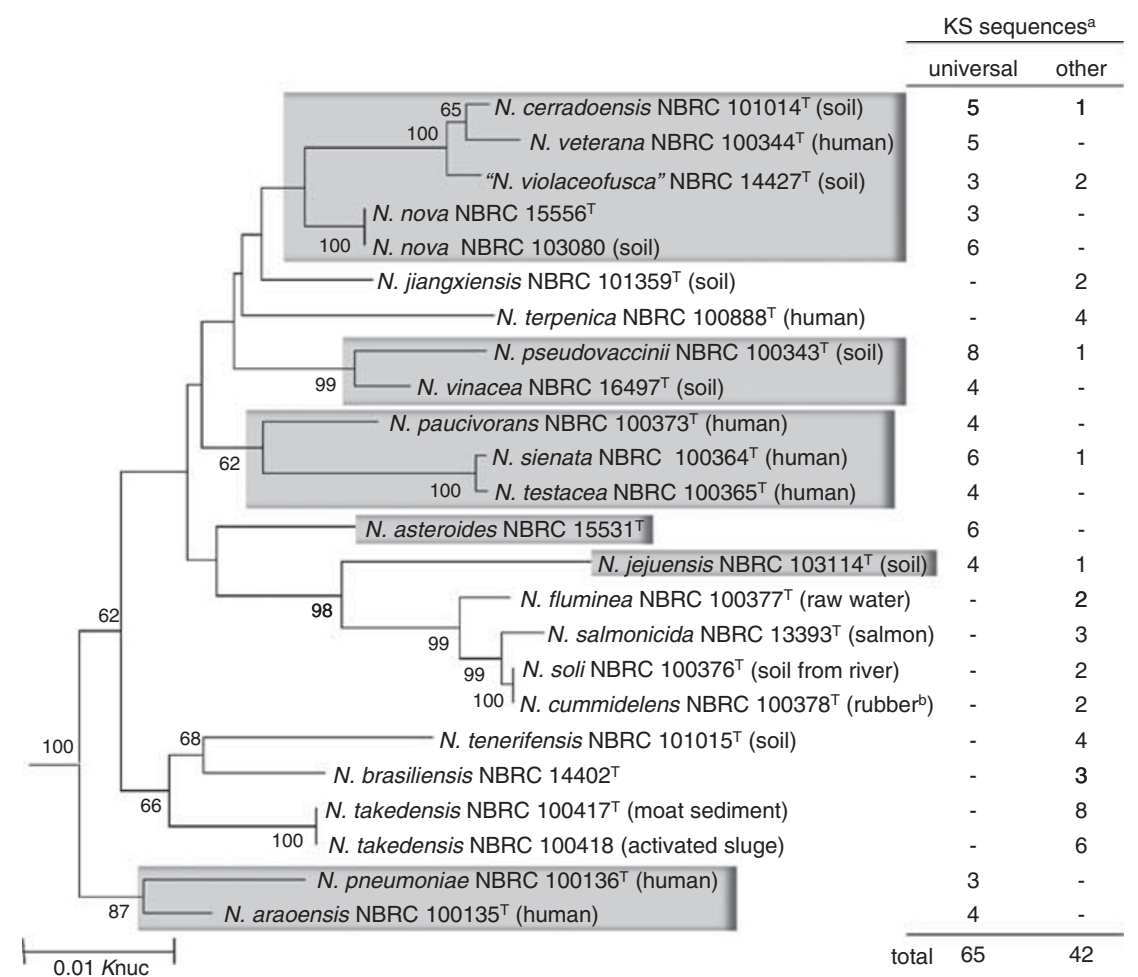

Figure $1 \mathrm{~A}$ phylogenetic tree of the test strains based on almost complete 16S rDNA sequences. Bootstrap values above 50\% from 1000 re-samplings are shown at the nodes. Strains shaded in gray possess universal PKS-I genes. The origins of the isolates are shown in parentheses. The origins of $N$. nova, $N$. asteroides and $N$. brasiliensis were not described by Tsukamura, ${ }^{21}$ Blanchard ${ }^{22}$ or Pinoy. ${ }^{23}$ aNumbers of non-redundant KS sequences obtained. The sequences shaded in Figure 2 and those in the others were counted. -, not detected. bVulcanized natural rubber pipe rings suspended in partially treated water from the River Thames. ${ }^{17}$

a phylogenetic tree (Figure 2). Strains identified as the same species, such as N. nova NBRC $15556^{\mathrm{T}}$ and N. nova NBRC 103080 or N. takedensis NBRC $100417^{\mathrm{T}}$ and N. takedensis NBRC 100418, contained almost identical PKS-I gene pairs. N. fluminea, N. salmonicida, N. soli and N. cummidelens, known as the N. salmonicida clade members, ${ }^{17}$ shared some common PKS-I genes. Likewise, phylogenetically close species such as $N$. cerradoensis and ' $N$. violaceofusca' also shared some common PKS-I genes. The 65 sequences from 14 strains, listed in Figure 1, could be classified into two groups as shown in Figure 2. The 65 sequences showed high sequence similarities (average $87 \%$ identity) to already published Nocardia KS sequences, suggesting that they are widely distributed among Nocardia spp. As different strains shared the KS sequences clustered in the same phylogenetic clade, it is suggested that they also shared the same type of PKS-I gene cluster involved in the synthesis of analogous metabolites. ${ }^{18}$ Therefore, we speculate that the polyketide compounds corresponding to the genes grouped in each clade shown in Figure 2 are analogous metabolites. To analyze the relationship between taxonomy and distribution of these PKS-I genes, a phylogenetic tree based on the $16 \mathrm{~S}$ rDNA sequences was constructed (Figure 1). The PKS genes grouped in Figure 2 were widely observed in the species belonging to certain clades. In contrast, strains lacking such universal PKS-I genes often possessed fairly diverse KS sequences. Among the 42 sequences that were not common, 36 were found in the strains lacking universal PKS genes (Figure 1). The KS sequences derived from $N$. brasiliensis, $N$. terpenica and $N$. takedensis were not observed in other species (Figure 2), suggesting that their PKS-I genes were unique. The species harboring such unique PKS genes were mainly located near N. brasiliensis and N. salmonicida in the $16 \mathrm{~S}$ rDNA phylogenetic tree. $N$. terpenica and $N$. jiangxiensis were phylogenetically distant from these; however, the bootstrap values were less than $50 \%$ and the topology was unstable. To interpret the relationship between PKS-I content and the taxonomy of the strains more precisely, reliable and high-resolution methods such as multilocus sequence typing should be carried out.

Recent genetic analyses of biosynthesis pathways for secondary metabolites have demonstrated their crucial roles in the discovery of novel natural products. Ayuso-Sacido and Genilloud ${ }^{13}$ examined the distribution of PKS-I genes in Nocardia strains using a PCR assay, and found that $69 \%(11 / 16)$ of the strains were positive for the presence of PKS-I genes. This was comparable to that in Streptomyces (79\%, 26/ 33); however, sequence diversity in Nocardia has not been thoroughly examined. ${ }^{12}$ We demonstrated that all the Nocardia strains tested in

Figure 2 A phylogenetic tree of the KS domain (approximately 366 amino acids) sequences in PKS-I genes of Nocardia species. Bootstrap values above $50 \%$ from 1000 re-samplings are shown at the nodes. PKS-I genes (gray) were universal in the 13 species highlighted in Figure 1. PKS-I genes in N. farcinica IFM 10152 were used as an outgroup because they are in a mixed PKS/NRPS gene cluster or in non-multimodular clusters whose KS domains are phylogenetically distinct. ${ }^{19,20 *}$ Indicates different strains identified as the same species that shared almost identical genes. ${ }^{* *}$ Indicates that phylogenetically close species shared similar genes. 


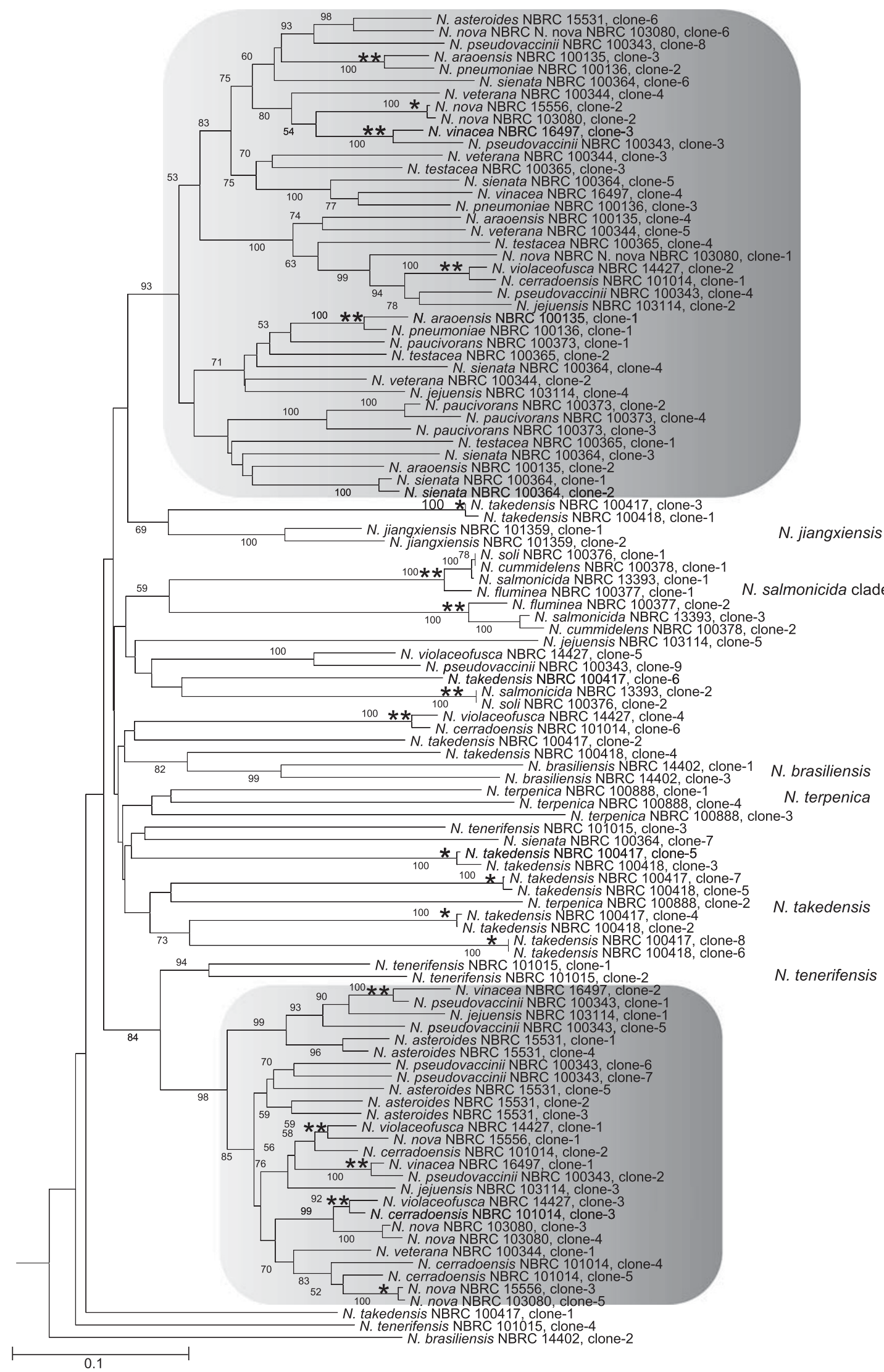


this study contained PKS-I genes and the diversity varied among taxonomic species. The 13 species possessed mainly universal PKS-I genes. Although the KS sequences were grouped into two clades, as shown by the phylogenetic analysis, the relationship between the two groups is still unclear from this study. We are sequencing the fulllength PKS gene cluster(s) in some strains to clarify this relationship. In contrast, the universal PKS-I genes were not detected in the other nine species shown in Figure 1. The sequences of KS domains in these nine species were different $(<70 \%$ identity) from the universal sequences and each other. Therefore, these species will be useful in screening sources for discovering novel types of polyketide compounds. As mentioned previously, N. brasiliensis and N. terpenica are known to be a useful source of various bioactive compounds. In this study, we revealed that some species, including $N$. brasiliensis and $N$. terpenica, had unique and diverse PKS-I genes. In contrast, reports of type-I polyketide products from other species are limited. ${ }^{2,8,9}$ This might be due to the poor PKS-I diversity in some species, in which universal PKS-I genes are dominant. It would be valuable to extensively analyze PKS-I genes in Nocardia strains and determine any relationship with their taxonomy.

\section{NUCLEOTIDE ACCESSION NUMBERS}

Accession numbers of the DNA sequences in Supplementary Table 1 are $\mathrm{AB} 606044-\mathrm{AB} 606150$.

1 Mikami, Y. Biological work on medically important Nocardia species. Actinomycetologica 21, 46-51 (2007)

2 Hoshino, Y. et al. Identification of nocobactin NA biosynthetic gene clusters in Nocardia farcinica. J. Bacteriol. 193, 441-448.

3 Nemoto, A. et al. Asterobactin, a new siderophore group antibiotic from Nocardia asteroides. J. Antibiot. (Tokyo) 55, 593-597 (2002).

4 Tanaka, Y. et al. Brasilinolide A, a new macrolide antibiotic produced by Nocardia brasiliensis: producing strain, isolation and biological activity. J. Antibiot. (Tokyo) 50, 1036-1041 (1997).

5 Mikami, Y. et al. A new antifungal macrolide component, brasilinolide B, produced by Nocardia brasiliensis. J. Antibiot. (Tokyo) 53, 70-74 (2000).
6 Hoshino, Y. et al. Nocardia terpenica sp. nov., isolated from Japanese patients with nocardiosis. Int. J. Syst. Evol. Microbiol. 57, 1456-1460 (2007).

7 Mikami, Y. et al. Production of erythromycin E by pathogenic Nocardia brasiliensis. J. Antibiot. (Tokyo) 52, 201-202 (1999).

8 Igarashi, M. et al. Tubelactomicin A, a novel 16-membered lactone antibiotic, from Nocardia sp. I. Taxonomy, production, isolation and biological properties. J. Antibiot. (Tokyo) 53, 1096-1101 (2000)

9 Ishikawa, J. et al. The complete genomic sequence of Nocardia farcinica IFM 10152. Proc. Natl. Acad. Sci. USA 101, 14925-14930 (2004).

10 Ohnishi, Y. et al. Genome sequence of the streptomycin-producing microorganism Streptomyces griseus IFO 13350. J. Bacteriol. 190, 4050-4060 (2008).

11 Omura, S. et al. Genome sequence of an industrial microorganism Streptomyces avermitilis: deducing the ability of producing secondary metabolites. Proc. Natl. Acad. Sci. USA 98, 12215-12220 (2001).

12 Ayuso, A. et al. A novel actinomycete strain de-replication approach based on the diversity of polyketide synthase and nonribosomal peptide synthetase biosynthetic pathways. Appl. Microbiol. Biotechnol. 67, 795-806 (2005).

13 Ayuso-Sacido, A. \& Genilloud, O. New PCR primers for the screening of NRPS and PKS-I systems in actinomycetes: detection and distribution of these biosynthetic gene sequences in major taxonomic groups. Microb. Ecol. 49, 10-24 (2005).

14 Khan, S. T. et al. Streptomyces associated with a marine sponge Haliclona sp.; biosynthetic genes for secondary metabolites and products. Environ. Microbiol. 13, 391-403 (2011).

15 Komaki, H. et al. Discovery of a pimaricin analog JBIR-13, from Streptomyces bicolor NBRC 12746 as predicted by sequence analysis of type I polyketide synthase gene. Appl. Microbiol. Biotechnol. 83, 127-133 (2009).

16 Tamura, K., Dudley, J., Nei, M. \& Kumar, S. MEGA4: molecular evolutionary genetics analysis (MEGA) software version 4.0. Mol. Biol. Evol. 24, 1596-1599 (2007).

17 Maldonado, L., Hookey, J. V., Ward, A. C. \& Goodfellow, M. The Nocardia salmonicida clade, including descriptions of Nocardia cummidelens sp. nov., Nocardia fluminea sp. nov. and Nocardia soli sp. nov. Antonie Van Leeuwenhoek 78, 367-377 (2000).

18 Jenke-Kodama, H., Borner, T. \& Dittmann, E. Natural biocombinatorics in the polyketide synthase genes of the actinobacterium Streptomyces avermitilis. PLoS Comput. Biol. 2, e132 (2006).

19 Jenke-Kodama, H., Sandmann, A., Muller, R. \& Dittmann, E. Evolutionary implications of bacterial polyketide synthases. Mol. Biol. Evol. 22, 2027-2039 (2005).

20 Moffitt, M. C. \& Neilan, B. A. Evolutionary affiliations within the superfamily of ketosynthases reflect complex pathway associations. J. Mol. Evol. 56, 446-457 (2003).

21 Tsukamura, M. Numerical analysis of the taxonomy of nocardiae and rhodococci. Division of Nocardia asteroides sensu stricto into two species and descriptions of Nocardia paratuberculosis sp. nov. Tsukamura (formerly the Kyoto-I group of Tsukamura), Nocardia nova sp. nov. Tsukamura, Rhodococcus aichiensis sp. nov. Tsukamura, Rhodococcus chubuensis sp. nov. Tsukamura, and Rhodococcus obuensis sp. nov. Tsukamura. Microbiol. Immunol. 26, 1101-1119 (1982).

22 Blanchard, R. Parasites vegetaux a l'exclusion des bacteries. in Traite de pathologie generale (ed. Bouchard, C.) vol. 2, 811-932 (G. Masson, Paris, 1896).

23 Pinoy, E. Actinomycoses et Mycetomes. Bull. Inst. Pasteur 11, 929-976 (1913).

Supplementary Information accompanies the paper on The Journal of Antibiotics website (http://www.nature.com/ja) 